\title{
The August 24, 2002 coronal mass ejection: when a western limb event connects to earth
}

\author{
Noé Lugaz ${ }^{1}$, Ilia I. Roussev ${ }^{1}$ and Igor V. Sokolov ${ }^{2}$ \\ ${ }^{1}$ Institute for Astronomy, University of Hawaii, 2680 Woodlawn Dr., Honolulu, HI, 96822, USA \\ email: nlugaz@ifa.hawaii.edu, iroussev@ifa.hawaii.edu \\ ${ }^{2}$ Department of AOSS, University of Michigan, 2455 Hayward St., Ann Arbor, MI 48198 \\ email: igorosk@umich.edu
}

\begin{abstract}
We discuss how some coronal mass ejections (CMEs) originating from the western limb of the Sun are associated with space weather effects such as solar energetic particles (SEPs), shocks or geo-effective ejecta at Earth. We focus on the August 24, 2002 coronal mass ejection, a fast $\left(\sim 2000 \mathrm{~km} \mathrm{~s}^{-1}\right)$ eruption originating from W81. Using a three-dimensional magneto-hydrodynamic simulation of this ejection with the Space Weather Modeling Framework (SWMF), we show how a realistic initiation mechanism enables us to study the deflection of the CME in the corona and the heliosphere. Reconnection of the erupting magnetic field with that of neighboring streamers and active regions modify the solar connectivity of the field lines connecting to Earth and can also partly explain the deflection of the eruption during the first tens of minutes. Comparing the results at $1 \mathrm{AU}$ of our simulation with observations by the $A C E$ spacecraft, we find that the simulated shock does not reach Earth, but has a maximum angular span of about $120^{\circ}$, and reaches $35^{\circ}$ West of Earth in 58 hours. We find no significant deflection of the CME and its associated shock wave in the heliosphere, and we discuss the consequences for the shock angular span.
\end{abstract}

Keywords. Sun: coronal mass ejections (CMEs), solar-terrestrial relations, acceleration of particles

\section{Introduction}

\subsection{The Eruption on August 24, 2002}

On August 24, 2002, active region (AR) 10069 was near the western limb of the Sun (W81) when it produced a powerful (X3.1) flare associated with a fast and wide coronal mass ejection (CME). This event has been well studied due to extensive remote observations by SoHO/LASCO and SoHO/UVCS (Raymond et al. 2003), in-situ by the Wind and $A C E$ spacecraft and also its inclusion as one of the Solar Heliospheric Interplanetary Environment (SHINE) campaign events. Based on LASCO observations, this was a wide and fast CME, with an average speed of $1,900 \mathrm{~km} \mathrm{~s}^{-1}$ within the first 20 solar radii. This event has been mostly studied in association with another wide and fast CME on April 21, 2002 for its association with a large Solar Energetic Particle (SEP) event (Tylka et al. 2005, 2006). Based on an increase of the iron-to-oxygen ration at large energy, these authors proposed that the shock geometry explain this difference: quasi-perpendicular for the August 24 and quasi-parallel for the April 21 one. The presence or absence of a reflecting boundary at or slightly ahead of Earth associated with a previous eruption has also been recently proposed to explain these differences (Tan et al. 2008).

\subsection{Studying Western-Limb Ejections}

Western limb events such as the August 24 and April 21, 2002 CMEs present a number of challenges for space weather prediction. Due to the Parker spiral, the Earth is on 
average magnetically connected with regions at the solar surface around W55 (for a $400 \mathrm{~km} \mathrm{~s}^{-1}$ background wind). Therefore, SEP events are preferentially associated with western events. Events such as the April 21, 2002 and August 24, 2002 present additional challenges since the SEP arrival time at Earth corresponds to a particle release height of less than $5 R_{\odot}$. If one believes that these particles are accelerated by the CME-driven shock wave, there are a number of scenarios to explain the observations. First, the shock must have formed low in the corona, and then, within $5 R_{\odot}$ of the solar surface, it either spaned at least $60^{\circ}$, or must have been significantly deflected towards the east, or the magnetic field line connecting Earth to the solar surface significantly diverged from the nominal Parker's spiral. Such differences of up to $30^{\circ}$ during SEP events between flare sites and the magnetic footpoint of the Earth on the solar surface have been reported before (Ippolito et al. 2005).

Western limb events are often associated with shocks and sometimes ejecta at 1 AU. Both August 24 and April 21, 2002 were associated with a shock wave at 1 AU which transited in about $55 \mathrm{hrs}$. Among large geomagnetic storms (Dst $\leqslant-100 \mathrm{nT}$ ) from the past solar cycle (Zhang et al. 2008), at least 6 were caused by a shock at Earth associated with an ejection western of W73. This fact, again, seems to imply either a very large span of the shock wave, a large deflection of the CME, or a combination of both.

Until now, it has been hard to study observationally the deflection of a CME in the corona or in the heliosphere due to the paucity of observations, especially in the nearEarth environment. Tripathi et al. (2004) reported deflections up to $20^{\circ}$ within the first hour after the initiation of an eruption based on a series of LASCO images. Shock span can also be estimated with white-light images, but it is limited by assumptions of symmetry and geometrical effects (Cremades et al. 2006). In these two examples, the determination of the CME span and deflection can be only be made for limb CMEs and only in the meridional direction. Additionally, the longitudinal extent of shocks can be estimated from multiple-spacecraft measurements; but until the launch of STEREO, it could only be done with spacecraft at different heliospheric distances, for example by the Helios spacecraft in the 1980s (DeLucas et al. 2008). The launches of STEREO and SMEI have also made 3-D tomography of CMEs easier (Jackson \& Hick 2002). On the theoretical and numerical sides, previous studies have focused on the deflection of a CME in the heliosphere due to its interaction with the solar wind (Wang et al. 2004). Here, based on a 3-D numerical simulation of the August 24, 2002 event, we discuss how, by using a new and realistic model of solar eruptions associated with a realistic model of the coronal magnetic field, we can study these different effects.

\section{Simulation Setup}

\subsection{Numerical Domains}

In our numerical model, the steady-state solar corona and solar wind are constructed following the methodology of Roussev et al. (2003), further described in Roussev et al. (2007). The initial condition for the coronal magnetic field is calculated by means of potential field extrapolation, following Altschuler et al. (1977), with boundary conditions for the radial magnetic field at the Sun, $B_{R}$, provided by full-disk SoHO/MDI observations taken four days before the eruption when the AR was closer to the disk center and better observed. The "solar" boundary in our model is placed at a height of $0.1 R_{\odot}$ above the photosphere. The plasma parameters are prescribed in an ad-hoc manner, through a variable polytropic index, to mimic the physical properties of streamers and coronal holes once a steady-state (non-potential) is reached. 
The time-dependent MHD equations for a single compressible fluid are solved using the Space Weather Modeling Framework (Tóth et al. 2005) using two physical domains: the Solar Corona (SC): $\{-20 \leqslant x \leqslant 20,-20 \leqslant y \leqslant 20,-20 \leqslant z \leqslant 20\} R_{\odot}$ and Inner Heliosphere (IH): $\{-220 \leqslant x \leqslant 220,-220 \leqslant y \leqslant 220,-220 \leqslant z \leqslant 220\} R_{\odot}$. We prescribe the initial grid in such a way as AR 10069 is resolved with cells as small as $4.9 \times 10^{-3} R_{\odot}$, 4 times finer than the rest of the solar surface. Additionally, the radial direction above the active region (resp. direction of the field lines connecting to Earth) is refined with cells of $0.08 R_{\odot}$ up to 8 (resp. 5) $R_{\odot}$ and $0.16 R_{\odot}$ up to 14 (resp. 10) $R_{\odot}$. The total number of computational cells is of the order of 1.77 and 14.66 millions, with largest meshes of size $1.25 R_{\odot}$ and $3.44 R_{\odot}$ for $\mathrm{SC}$ and $\mathrm{IH}$, respectively.

To the initial magnetic field constrained by SoHO/MDI data, we superimposed newly emerged magnetic flux simulated by a dipolar magnetic field of two point charges. These two charges are initially separated by $5 \times 10^{3} \mathrm{~km}$ and buried at a depth of $3 \times 10^{4} \mathrm{~km}$ under the solar surface, and chosen so that the peak value of the radial magnetic field at the solar surface is about 47 Gauss.

\subsection{Solar Eruption Model}

To initiate the eruption, we use the model described in Roussev et al. (2007). To summarize, once the steady-state is reached at $t=0$, the two magnetic charges are moved apart quasi-steadily up to $t=t_{S}=20 \mathrm{~min}$ with a speed which is ramped up in $t_{S} / 3$ to $80 \mathrm{~km} \mathrm{~s}^{-1}$; the charge motion is stopped at $t=t_{S}$.

In addition to updating the radial component of the dipole field at the boundary, we also impose the accompanying horizontal boundary motions. As the result of moving the charges apart, the magnetic field lines connecting the two spots of the dipole are stretched. With appropriate choice of parameters describing the relative position of the charges, their strength and speed of motion, one can achieve a quasi-steady magnetic field evolution toward a state that is no longer stable. Then, as the result of loss of confinement with the overlying field, the "energized" magnetic field of the dipole erupts, manifesting as a CME.

\section{Eruption and Coronal Evolution}

\subsection{Loss of Equilibrium}

One of the main results of the work in Roussev et al. (2007) was to recognize the importance of the pre-existing magnetic topology in the initiation of the eruption. This is first and foremost because reconnection at the pre-existing null points and quasi-separatrix layers (QSLs) enables the sheared and energized magnetic flux of the dipole to erupt. This work was the continuation and adaptation to realistic background coronal magnetic fields of previous work by Antiochos et al. (1999); Galsgaard et al. (2005) and Pontin \& Galsgaard (2007). There are two main pre-existing topological features important to understand this eruption: a null point between ARs 10067 and 10069 and a QSL between ARs 10066, 10068 and 10069 (the magnetic topology before the shearing phase is illustrated in the left panel of Figure 1). Noteworthy is the fact that there are open field lines originating from AR 10069 and AR 10067 which pass in proximity of the null point.

During the shearing, current builds up along the loops connecting the two magnetic spots of the dipole and the magnetic field lines expand until they reconnect with the overlying field through the QSL. The QSL is disrupted and becomes a current sheet which starts erupting (as illustrated in the right panel of Figure 1). The erupting field lines are now connecting AR 10069 to ARs 10067 and 10066. As some of these field lines expand further, they reconnect though the null point and some of them open up. The 

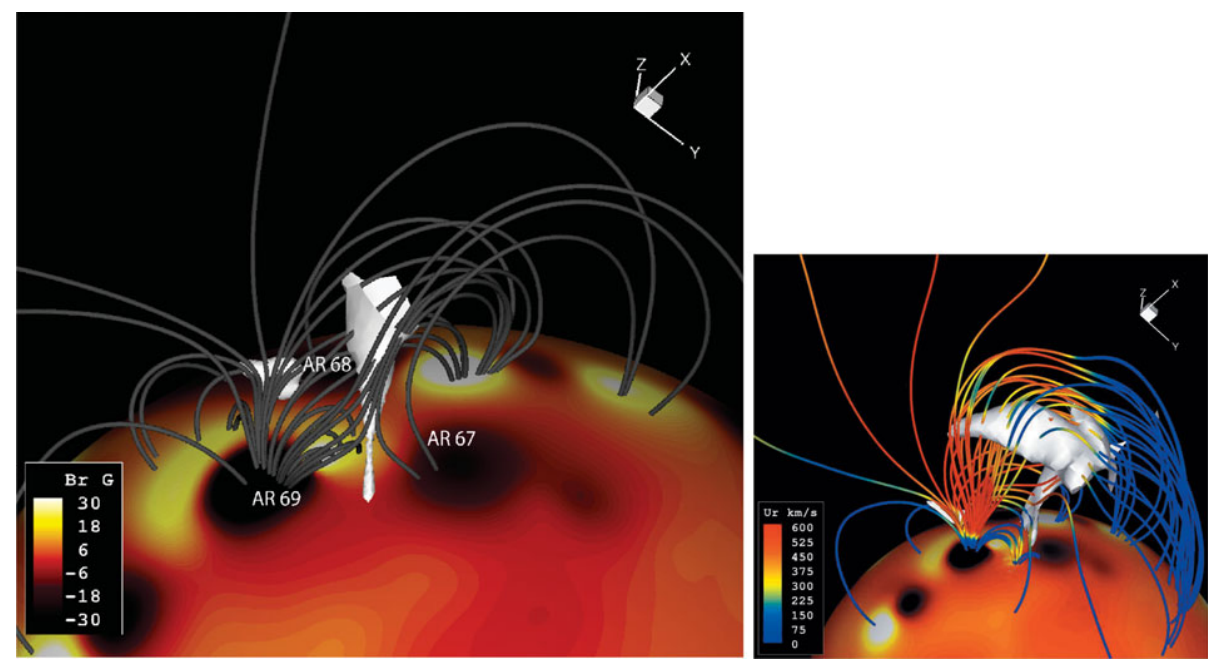

Figure 1. Magnetic topology before (left) and after (right, $t=20 \mathrm{~min}$ ) the shearing phase. The solar surface is color-coded with the radial magnetic field strength and the magnetic field lines with the radial velocity for the right panel. The null point and quasi-separatrix layer (QSL) are visualized as white isosurfaces of plasma beta equal to 0.15 (left) and 0.4 (right).

main motion of the erupting flux is radially above the initial position of the QSL but the reconnection through the null point between ARs 10069 and 10067 also enables the expansion of the CME towards the east (Earth direction).

\subsection{Magnetic Connection to Earth}

The August 24, 2002 eruption was associated with a large SEP event, even though the magnetic connectivity to Earth is hard to assess due to the position of the AR $81^{\circ}$ west of disk center. The timing of the arrival of SEPs at Earth is consistent with particle release at less than $5 R_{\odot}$. Additionally, contrary to our model which is not potential, the potential field source surface model (Altschuler et al. 1977) shows that there are no open magnetic field lines originating from AR 10069. Our simulation can help explain why a SEP event was indeed observed at Earth. We will not focus on a given field line, but on a set of field lines spanning about $10^{\circ}$ at 1 AU including the one connected to Earth on August 24, 2002 01UT. A number of reasons make the determination of the exact footpoint of the field line connected to Earth difficult and compelled us to consider a stack of field lines instead. First, the solar magnetic field is reconstructed from observations on August 21, 2002, 3 days prior to the studied event and the coronal and photospheric magnetic fields have certainly changed in this time span. Second, as noted by Tan et al. (2008), the presence of prior ejections in the heliosphere may significantly modify the magnetic connectivity. There was a number of ejections prior to the August 24 ones, alhough there was no clear ejecta passing Earth at this time according to satellite data. Random walk of the field lines may also account for up to $10^{\circ}$ longitudinal variation on the solar surface (Ippolito et al. 2005). Last, as can be seen on the left panel of Figure 3, field lines connecting to the vicinity of Earth before the start of the shearing phase have footprints in two very distinct zones on the solar surface through a QSL: one around N20W70 and one around S15W10. The "average" position of the field lines connecting to Earth is about W40, indeed not departing too much from the nominal Parker's spiral. However, 

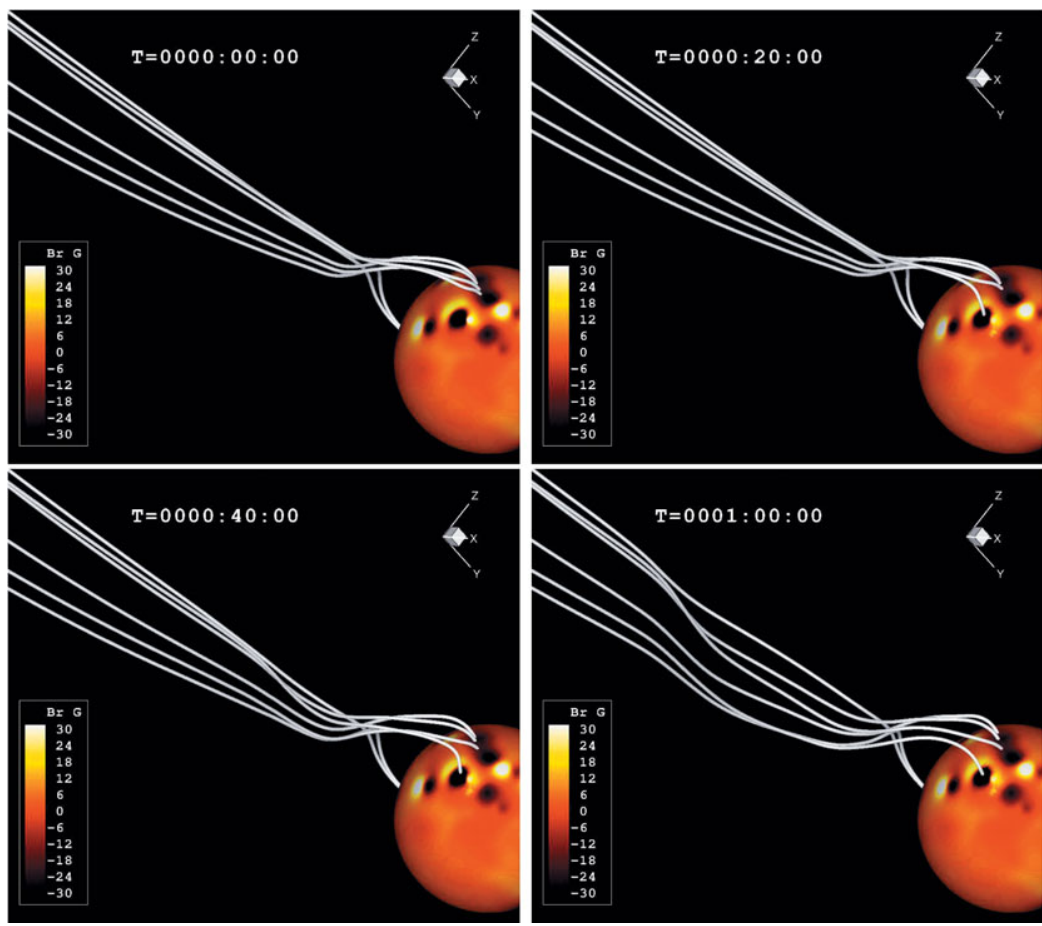

Figure 2. Field lines connecting to the vicinity of Earth and their evolution during the early phase of the eruption. The solar surface is color-coded with the radial magnetic field strength. Note the change of connectivity of one of the field lines at $t=20 \mathrm{~min}$ which connect AR 10069 to Earth. Note also the propagation of the shock wave along the field lines at later times.

the magnetic topology close to the Sun is such that some of these field lines connect to the proximity of AR 10069 .

As described above, we find that, by the end of the shearing phase, the erupting flux has reconnected with open magnetic field lines from AR 10067 through the null point and some erupting field line are now direclty connected to Earth's vicinity. This can be seen in the top right panel of Figure 2. The disruption of these field lines due to the passage of the shock wave can be seen at the later times. We find that the shock wave has been formed by $5 R_{\odot}$. It has also been significantly deflected due to reconnection at the northern null point. An approximate visualization of the shock wave can be seen on the right panel of Figure 3 along with the field lines connecting to Earth's vicinity. We should also note that the shock does not become quasi-parallel along Earth-connected field lines until about 90 minutes after the start of the eruption (see also Roussev et al. 2008). A similar evolution of the shock angle (from quasi-perpenicular to quasi-parallel) with distance has been previously reported in Manchester et al. (2005) for a field line about $37^{\circ}$ north of the center of the CME.

\section{Heliospheric Evolution and Results at 1 AU}

A shock wave associated with the eruption of August 24 was detected at Earth by $A C E$ about 58 hours after its start. In our simulation, the shock wave does not extend all the way to Earth. In fact, we find that the shock wave reaches 1 AU approximatively 42 hours after the end of the shearing phase about $60^{\circ}$ West of Earth. Its maximum angular 

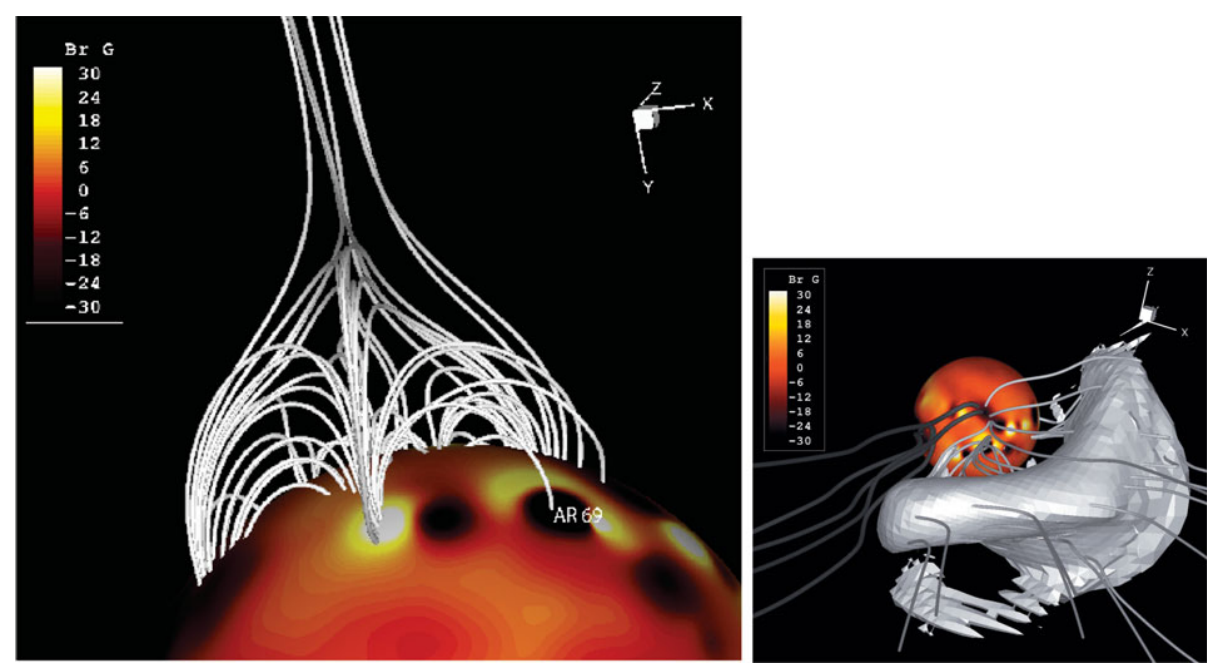

Figure 3. Left: Solar footpoints of the magnetic field lines connecting to the vicinity of Earth, which span from S15W05 to N20W75. Right: Visualization of the shock wave 1 hour after the start of the shearing phase. Field lines connecting to Earth's vicinity are shown with darker grey and larger radii. The white surface is an isosurface of Aflvénic Mach equal to 1.
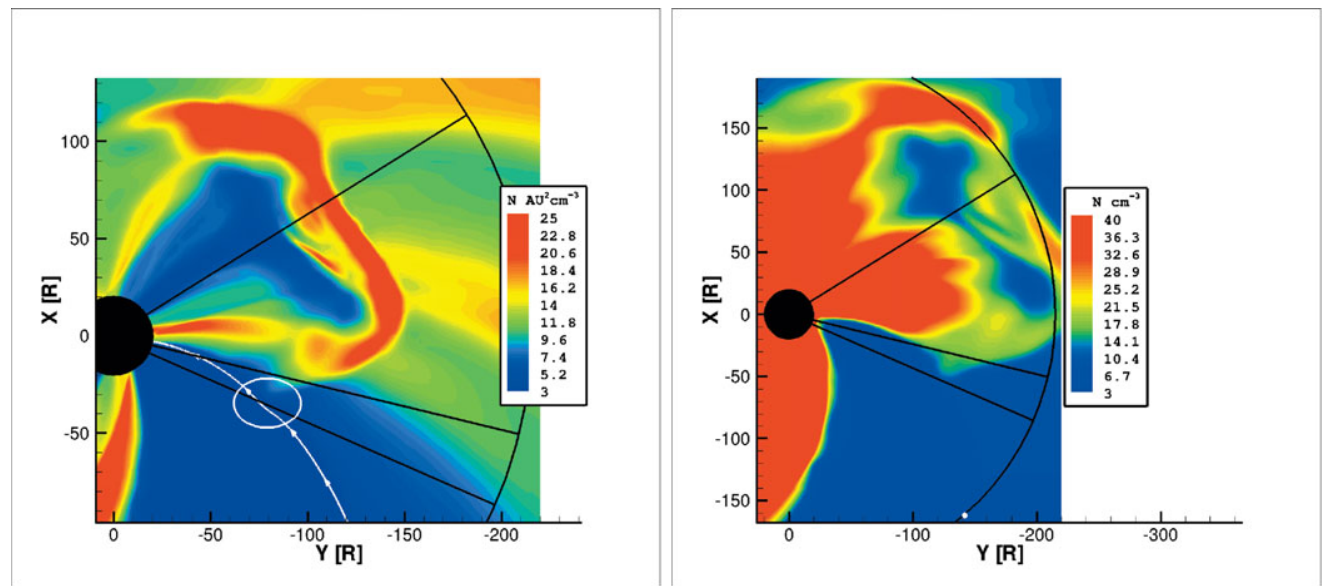

Figure 4. Number density (left, scaled by $1 / \mathrm{R}^{2}$ ) in the equatorial plane 29 hours (left) and 48 hours (right) after the start of the shearing phase. The black circle represent Earth's orbit, Earth's position is shown with a white disk. The three lines are, from top to bottom, the radial directions corresponding to the central position of the eruption, the longitude reached at 58 hours and the maximum longitudinal extent. The field line connecting to Earth is shown in white and the shock position is highlighted with the white ellipse.

span is about $120^{\circ}$ and it reaches a maximum of $25^{\circ}$ West of Earth. After 58 hours, the simulated shock wave reaches a point about $35^{\circ}$ West of Earth. The results at 48 hours are shown on the right panel of Figure 4.

In agreement with previous studies (e.g. Jacobs et al. 2007), we find that there is no significative non-radial expansion of the CME in the heliosphere past the upper corona. Although in our simulation the shock wave does not hit Earth, we find that there is direct magnetic connection between the flank of the shock and Earth during most of the heliospheric evolution of the CME and its associated shock. This is illustrated at time 29 
hours in the left panel of Figure 4 and may have important consequences to understand and predict the observed Forbush decrease of ACRs (Eroshenko et al. 2008) and the time variation of the SEP event.

\section{Discussions and Conclusion}

We performed a Sun-to-Earth simulation of the August 24, 2002 CME event with a realistic CME initiation mechanism (Roussev et al. 2007). This is one of the first solar-terrestrial simulation involving a CME model more complex than a flux rope or a simple perturbation added onto the solar wind. Using a realistic model is required to study space weather effect such as (i) the formation of the shock wave, (ii) the change of connectivity between the Earth and the solar surface during the eruption, and (iii) the possible deflection of the CME. We find that reconnection of the erupting flux at one null point north-east of the active region results in the deflection of the eruption in the corona (we find no subsequent deflection in the heliosphere) towards the Sun-Earth line. There is an opening of part of the erupting flux due to this reconnection event, and, consequently, a change of magnetic connectivity with Earth. We find that the shock wave has formed by $5 R_{\odot}$ and has a sufficient longitudinal extent to accelerate particles along Earth-connected field lines.

Last, we must investigate why, in our simulation, the shock does not reach Earth. First, contrary to what was inferred by Wang et al. (2004), we find no consequent deflection of the CME in the heliosphere, even though it is a fast western CME. This might be because the solar wind is not well reproduced. Or it might be because there is in fact no significant deflection of $\mathrm{CME}$ in the heliosphere. If we believe that there is no large deflection in the heliosphere, then the shock angular extent must be at least $170^{\circ}$ to explain the detection of the shock wave by $A C E$, significantly larger than what is predicted by our model. It is worth noting that the simulated CME is slower in the corona than the observed one by as much as $35 \%$; a faster CME will most likely be associated with a larger shock wave. We will investigate this in future simulations of the same event. In situ observations by STEREO (and Helios) and future polar coronagraphs can valuable information concerning the angular extent of shock wave, and possibly a deflection.

\section{Acknowledgements}

The research for this manuscript was supported by NSF grants ATM0639335 and ATM0819653 and NASA grants NNX07AC13G and NNX08AQ16G.

\section{References}

Altschuler, M. D., Levine, R. H., Stix, M., \& Harvey, J. 1977, Solar Phys., 51, 345

Antiochos, S. K., DeVore, C. R., \& Klimchuk, J. A. 1999, ApJ, 510, 485

Cremades, H., Bothmer, V., \& Tripathi, D. 2006, Adv. Space Res., 38, 461

DeLucas, A. et al. 2008, in this volume

Eroshenko, E. et al. 2008, in this volume

Galsgaard, K., Moreno-Insertis, F., Archontis, V., \& Hood, A. 2005, ApJ Lett., 618, L153

Ippolito, A., Pommois, P., Zimbardo, G., \& Veltri, P. 2005, A \& A, 438, 705

Jackson, B. V. \& Hick, P. P. 2002, Solar Phys., 211, 345

Jacobs, C., van der Holst, B., \& Poedts, S. 2007, A \& A, 470, 359

Manchester, W. B., IV, et al. 2005, ApJ, 622, 1225

Pontin, D. I. \& Galsgaard, K. 2007, JGR, 112, 3103

Raymond, J. C., et al. 2003, ApJ, 597, 1106

Roussev, I. I., et al. 2003, ApJ, 595, L57 
Roussev, I. I., Lugaz, N., \& Sokolov, I. V. 2007, ApJ Letters, 668, L87

Roussev, I. I., Lugaz, N., \& Sokolov, I. V. 2008, AIP Conf.Ser., 1039, 286

Tan, L. C., Reames, D. V., \& Ng, C. K. 2008, ApJ, 678, 1471

Tóth, G., et al. 2005, J. Geophys. Res., 110, 12226

Tripathi, D. et al. 2004, Multi-Wavelength Investigations of Solar Activity, 223, 401

Tylka, A. J. et al. 2005, ApJ, 625, 474

Tylka, A. J. et al. 2006, ApJS, 164, 536

Wang, Y., Shen, C., Wang, S., \& Ye, P. 2004, Sol. Phys., 222, 329

Zhang, J., Poomvises, W., \& Richardson, I. G. 2008, Geophys. Res. Lett., 35, 2109 